\title{
BRCA1 Promoter Hypermethylationas an Early Diagnostic Tool for Breast Cancer
}

\author{
Tehseen Hassan ${ }^{1, ~}$, Showkat Ahmad Bhat ${ }^{1}$, Sabhiya Majid ${ }^{1}$, Manzoor R. Mir ${ }^{2}$, \\ Purnima Shrivastava ${ }^{3}$ \\ ${ }^{1}$ Department of Biochemistry, Govt. Medical College Srinagar, Jammu \& Kashmir, India \\ ${ }^{2}$ Division of Vety Biochemistry, Faculty of Veterinary Sciences, SKUAST-K, Jammu \& Kashmir, India \\ ${ }^{3}$ Director Research Bhagwant University, Sikar Road Ajmer, Rajasthan, India
}

Email address:

Khan.teh@gmail.com (T. Hassan)

${ }^{*}$ Corresponding author

\section{To cite this article:}

Tehseen Hassan, Showkat Ahmad Bhat, Sabhiya Majid, Manzoor R. Mir, Purnima Shrivastava. BRCA1 Promoter Hypermethylationas an Early Diagnostic Tool for Breast Cancer. Cancer Research Journal. Vol. 5, No. 2, 2017, pp. 9-13. doi: 10.11648/j.crj.20170502.11

Received: May 1, 2017; Accepted: May 24, 2017; Published: June 23, 2017

\begin{abstract}
Background: Breast cancer is the second most common cancer among women after cervical cancer. As Cancer development and progression is dictated by chain of alterations in genes. Over the past few years, the Kashmir valley has witnessed a tremendous increase in the incidence of breast cancer in its unexplored ethnic population. The aim of present study was to find out the role of Promoter Hypermethylation of BRCA1 gene in Breast cancer patients. Material Methods: The DNA was extracted from all the samples and was modified using bisulphite modification kit. Methylation-specific polymerase chain reaction was used for the analysis of the promoter hypermethylation status of BRCA1 gene. Results: The epigenetic analysis revealed that unlike other high risk regions, Kashmiri population has a different promoterhypermethylation profile of BRCA1 gene as $68 \%$ of the cases showed BRCA1 promoter hypermethylation in comparison to $20 \%$ of the normal cases, the association of promoter hypermethylation with breast cancer and normal cases was found to be significant $(\mathrm{P}=0.0006)$. The frequency of BRCA1 promoter hypermethylation was found to be certainly higher in Stage III/IV (75\%) compared to Stage I/ II $(62 \%)$ but the difference was not statistically significant $(\mathrm{P}=0.0674)$. The frequency of promoter methylation was foundhigher (77.1\%) in age group above 60- years) than ages below 60 years. Conclusion: These results suggest that BRCA1 aberrant promoter hypermethylation in Kashmiri population contributes to the process of carcinogenesis in breast cancer and is reportedly one of the commonest epigenetic changes in the development of breast cancer.
\end{abstract}

Keywords: Breast Cancer, BRCA1, Hypermethylation, Bisulphite Treatment

\section{Introduction}

The breasts are external symbol of beauty and womanhood in women; however cancer of the breast is responsible for the death of millions of women worldwide every year. Malignancy of the breast is one of the commonest causes of death in women aged between 40-45 years (Showkat, et al., 2013). The incidence of this disease is rising in many countries such as Japan and other developing nations and has become a genuine public health problem, with one woman in ten, developing it in her lifetime throughout the world. The incidence of breast cancer increases with age, being uncommon below the age of 32 years (Wernberg et al.,
2009). Epigenetics changes, as DNA methylation is one of the most common changes of epigenetics events, covalent addition of the methyl group to DNA, which played an important role in driving tumorgenesis (Fang., et al 2012). The DNA methylation usually occurs in the $\mathrm{CpG}$ islands located in or near the promoter of over $70 \%$ of all genes. In breast cancer, hypermethylation occurs in BRCA1 gene's promoter leaded to the cancer development and progression (Ramezani., et al., 2012 and Phuong et al., 2014). Three main features of BRCA1 are thought to be important for its function.BRCA1 has been implicated in many cellular 
functions including cell cycle regulation, DNA damage response, maintenance of genomic stability and recombination, amongstothers (Parvin et al., 2004). Aberrant DNA methylation is now recognized as one of the most common molecular abnormalities in cancer and references therein). Thisepigenetic modification occurs at the cytosines of $\mathrm{CpG}$ dinucleotides, which often exist in clusters called $\mathrm{CpG}$ islands. Methylation of these sites in the promoter region of a gene can result in chromatin condensation and gene silencing. In cancer cells, aberrant methylation has frequently been reported in tumor suppressor genes, DNA repair genes and genes related to cancer metastasis and invasion.In the present study, we analyze the hypermethylationstatus of BRCA1, in breast cancer patients and normal controls in ethenic population of Kashmir valley.

\section{Materials and Method}

\subsection{Sample Collection}

100 surgically obtained breast tissue Samples among which 75 were breast cancer patients and 25 were normal samples were obtained from the Department of Surgery, of Shri Maharaja Hari Singh (S. M. H. S) hospital Srinagar in sterilized plastic vials containing $10 \%$ of normal saline and transported from the theatres to the laboratory on ice and stored at $-80^{\circ} \mathrm{C}$ till further process. The information regarding gender, age stage, occupation and residence were collected from the record file of patients present in the hospital and histological grade for each sample was collected from the histopathological reports. The DNA was extracted by phenol chloroform method (Sam brook \& Russell). All breast cancer patients included in the study were females, with the histopathological diagnosis of the breast cancer.

\subsection{Qualitative and Quantitative Analysis of Genomic DNA}

The integrity of the genomic DNA was examined by gel electrophoresis using 1\% agarose gel. DNA in the gel was visualized with the help of gel doc system (Biorad). The quantity of DNA was determined by U.V. spectrophotometric method.

\subsection{Bisulpite Treatment}

Sodium bisulfite treatment converts unmethylatedcytosines to uracil and hence enabled to distinguish between the hypermethylated and non hypermethylated cytosine residues. DNA was modified by kit based method (EZ DNA Methylation $^{\mathrm{TM}}$ Kit) supplied by ZYMO RESEARCH. The modified DNA was stored at $-20^{\circ} \mathrm{C}$ for further use.

\subsection{Methylation Analysis}

Methyl specific polymerase chain reaction (MSP): Amplification of the promoter region of the gene was carried out in Eppendorf Gradient Thermalcycler in a $25 \mu$ l reaction mixture as shown in table 1 . Information about primers is given in table 2 .
Table 1. Volume and concentrations of different reagents used in PCR

\begin{tabular}{lll}
\hline Reagent & Concentration & Volume \\
\hline PCR MM (master mix) & & $12.5 \mu \mathrm{l}$ \\
Forward primer & $10 \mathrm{pmol} / \mu \mathrm{l}$ & $1 \mu \mathrm{l}$ \\
Reverse primer & $10 \mathrm{pmol} / \mu \mathrm{l}$ & $1 \mu \mathrm{l}$ \\
DNA sample & $250 \mathrm{ng} / \mu \mathrm{l}$ & $1 \mu \mathrm{l}$ \\
Deionised water & & $9.5 \mu \mathrm{l}$ \\
Total volume & & $25 \mu \mathrm{l}$ \\
\hline
\end{tabular}

Table 2. Primers described by Herman (25) used and length of fragments obtained in MSP (Methylation Specific PCR).

\begin{tabular}{lll}
\hline Nature of Primer & Primer sequence & \\
\hline unmethylatedPrime & Forward primer & 5'ttggttttgtggtaatggaaaagtgt3' \\
$(B R C A 1)$ & Reverse primer & 5'caaaaaatctcaacaaactcacacca ' \\
methylated & Forward primer & 5'tcgtggtaacggaaaagcgc3' \\
primer $(B R C A 1)$ & Reverse primer & 5'aaatctcaacgaactcacgccg3' \\
\hline
\end{tabular}

Gradient thermal cycler (Eppendorf) was programmed as in table: 3 , to carry out the PCR amplification.

Table 3. Thermal cycling conditions.

\begin{tabular}{llll}
\hline Steps & Temperature $^{\circ} \mathbf{C}$ & Time & $\begin{array}{l}\text { Number of } \\
\text { cycles }\end{array}$ \\
\hline 1. Initial Denaturation & 95 & $5 \mathrm{~min}$ & 1 \\
2. Denaturation & 95 & $30 \mathrm{sec}$ & \\
3. Annealing & $56.9 / 60.4$ & $30 \mathrm{sec}$ & 35 \\
4. Extension & 72 & $30 \mathrm{sec}$ & \\
5. Final extension & 72 & $4 \mathrm{~min}$ & 1 \\
6. Hold & 42 & $5 \mathrm{~min}$ & \\
\hline
\end{tabular}

After completion of PCR, the reaction products were run on $2 \%$ agarose gel, with ladder of $100 \mathrm{bp}$ as marker, methylated and unmathylated bands were counted in all samples.

\section{Statistical Analysis}

The data obtained was statistically assessed by descriptive analysis, one-way ANOVA using SPSS (SPSS Software products, Marketing Department, SPSS Inc. Chicago, USA).

\section{Results}

\subsection{Analysis of BRCA-1 Promoter Hypermethylation}

Figure-1 showed DNA isolated from case samples with phenol-chloroform method (Sambrook et al., 1989).

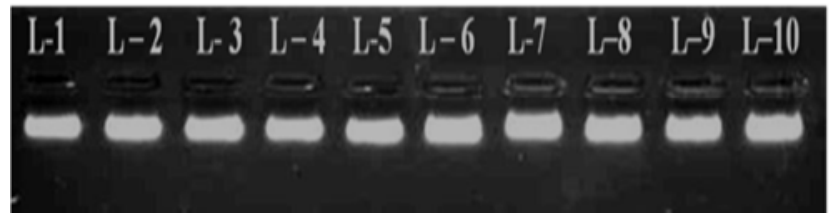

Figure 1. Lane (L) 1-10 showing the isolated DNA of case samples, run on 1\% Agarose gel. 


\subsection{Relationship of Promoter Methylation of BRCA-1 Gene with Breast Cancer in Histopathologically Confirmed Cancer and Normal Cases}

Methylation-specific PCR was done to examine the methylation status of the promoter region of BRCA-1gene figure $2 \& 3.68 \%(51 / 75)$ of the breast cancer tissue samples showed methylated BRCA-1promoter and 32\% (24/75) of the cases however showed unmethylated BRCA-1promoter (Table 4). Almost all 80\% (20/25) of the histopathologically confirmed normal tissue samples showed unmethylated BRCA- 1 promoter, except only in $20 \%$ cases $(4 / 20)$ where BRCA-1promoter was found to be methylated (graph-1 and graph-2). The association of promoter methylation with breast cancer was evaluated by $\chi^{2}$ (Chi square) test, using SPSS soft weare and was found to be significant $(\mathrm{P}=0.0006)$.

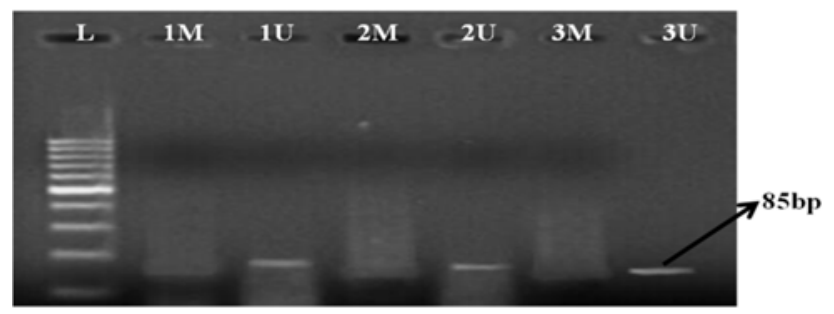

Figure 2. MSP (Methylation Specific PCR) of histopathologically confirmed Normal Breast DNA Samples Run on 2\% Agarose Gel.

L- 50bp marker

Cases amplified with unmethylated primer, product size was $85 \mathrm{bp}$.

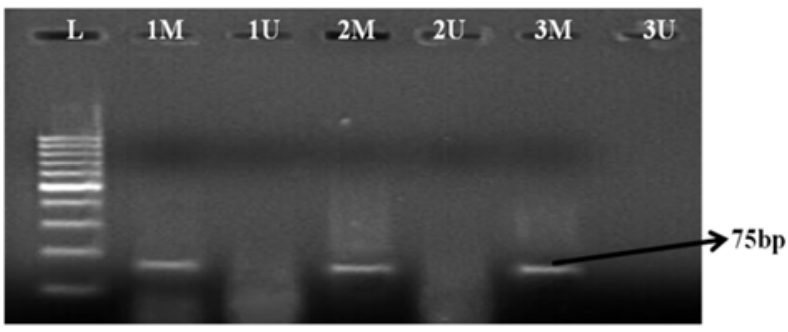

Figure 3. MSP (Methylation Specific PCR) of Breast Cancer DNA Samples Run on 2\% Agarose Gel. Methylated Product is of $75 \mathrm{bp}$ length and unmethyaletd is $85 \mathrm{bp}$ in length.

L - 50bp marker

Cases amplified with methylated primer, product size was $75 \mathrm{bp}$.

Table 4. Number \& Frequency of Methylated/Unmethylated Cancer \&Normal Cases.

\begin{tabular}{lll}
\hline Cancer cases (75) & & Frequency \\
\hline Methylated & 51 & $68 \%(51 / 75)$ \\
Unmethylated & 24 & $32 \%(24 / 75)$ \\
Normal samples (25) & & Frequency \\
Methylated & 5 & $20 \%(5 / 25)$ \\
Unmethylated & 20 & $80 \%(20 / 25)$ \\
\hline
\end{tabular}

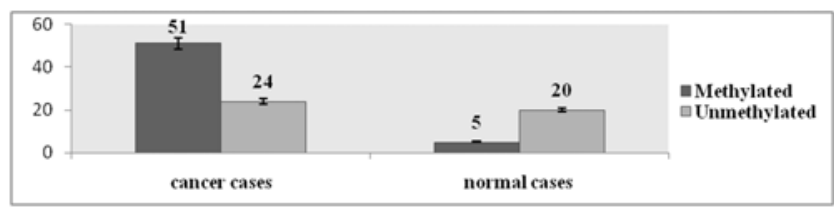

Graph 1. Number of Methylated/Unmethylated Cancer \& Normal Cases.

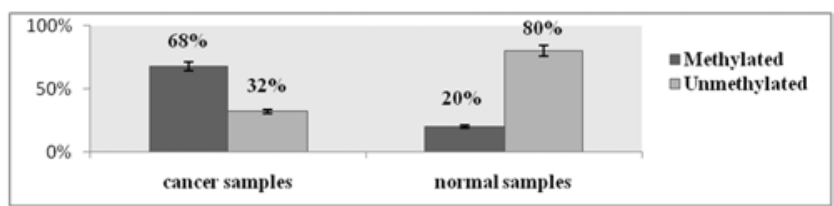

Graph 2. Frequency of Methylated/Unmethylated Cancer \& Normal Cases.

\subsection{Relationship Between Promoter Methylation of BRCA-1 Gene and Selected Clinico-Pathological Parameters: These Parameters Included Clinical Staging and Age}

\subsubsection{Relationship of Promoter Methylation of BRCA-1 Gene in Stage I/ II and Stage III/IV}

There were 43 breast cancer cases that were in Stage I and Stage II of the disease. Among these cases 27 cases were methylated and 16 cases were unmethylated. However, among 32 cases that were in Stage III and Stage IV of the disease, 24 cases were methylated and 8 cases were unmethylated (Table 5). When the frequency of $B R C A$ 1 promoter methylation was compared with clinical staging of the disease, $B R C A-1$ promoter methylation was found to be certainly higher in Stage III/IV (75\%) compared to Stage I/ II $(62.7 \%)$ but the difference was not statistically significant $(\mathrm{P}$ $=0.0674)$.

Table 5. Number of Cases Showing Promoter Methylation \&Unmethylation in Stage I/II \& Stage III/IV during MSP Amplification Confirmed By 2\% Agarose Gel Electrophoresis.

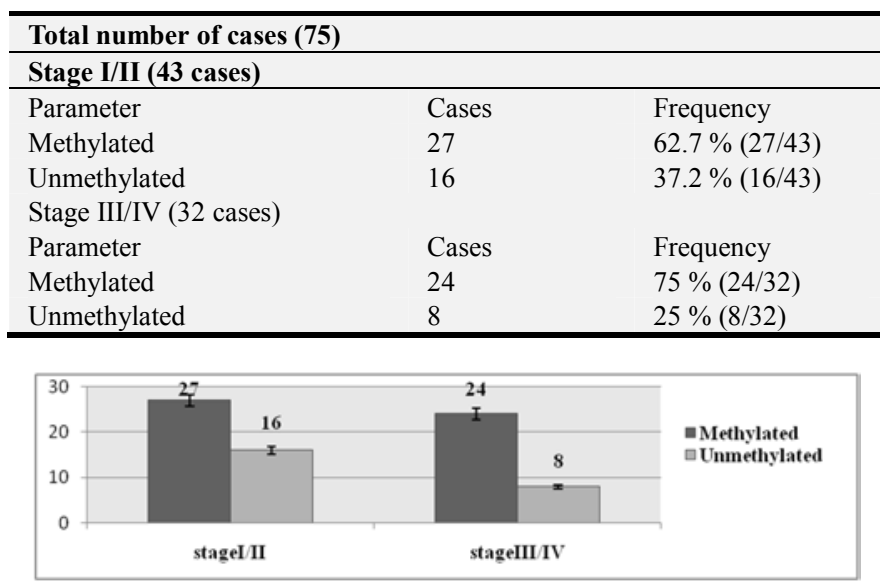

Graph 3. Number of Methylated \&Unmethylated Cases of Different Stages of Breast Cancer Cases.

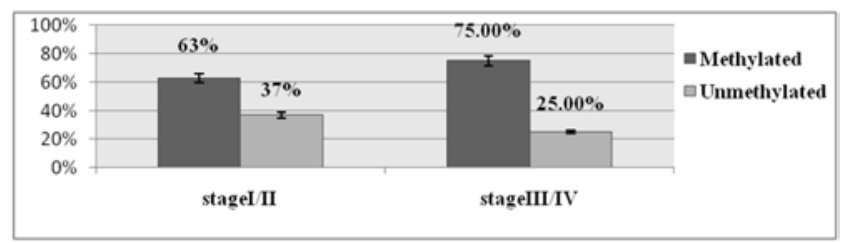

Graph 4. Frequency of Methylated \&Unmethylated Cases of Different Stages of Breast Cancer Cases.

\subsubsection{Relationship of Promoter Methylation in the Breast Cancer Cases and Normal Samples of Different Age Groups}

Among 75 breast cancer cases, 6 belonged to age group (0- 
30 yrs), 34 belonged to age group (31-60yrs) and 35 belonged to age group (61-90yrs). However the number of methylated cases in each age group was 2,22 and 27 respectively, as shown in Table 6 and graph $5 \& 6$. The frequency of promoter methylation was found $33.33 \%$ in age group (0-30 yrs), 64.7\% in age group (31-60 yrs) and $77.1 \%$ in age group (61-90) yrs) as shown in Table 6 and grapg 7. Similarly among 25 normal cases, 2 belonged to age group (0-30 yrs), 10 belonged to age group (31-60 yrs) and 13 cases belonged to age group (61-90 yrs). However the number of methylated cases in each of the age groups were 0,2 and 3 respectively (Table 6).The frequency of promoter Methylation was found $0 \%$ in age group (0-30yrs), 20\% in age group (31-60yrs) and $23 \%$ in age group (61-90yrs) as shown in graph 7.

Table 6. Methylation Pattern of Different Age Groups During MSP Amplification Confirmed By 2\% Agarose Gel Electrophoresis.

\begin{tabular}{lllll}
\hline & & $\mathbf{0 - 3 0}$ years & $\mathbf{3 1 - 6 0}$ years & $\mathbf{6 1 - 9 0}$ years \\
\hline \multirow{2}{*}{$\begin{array}{l}\text { Cancer } \\
\text { cases(40) }\end{array}$} & Total & 6 & 34 & 35 \\
& Methylation & 2 & 22 & 27 \\
& Frequency & $33.3 \%$ & $64.7 \%$ & $77.1 \%$. \\
Normal & Total & 2 & 10 & 13 \\
Cases(20) & Methylation & 0 & 2 & 3 \\
& Frequency & $0 \%$ & $20 \%$ & $23 \%$ \\
\hline
\end{tabular}

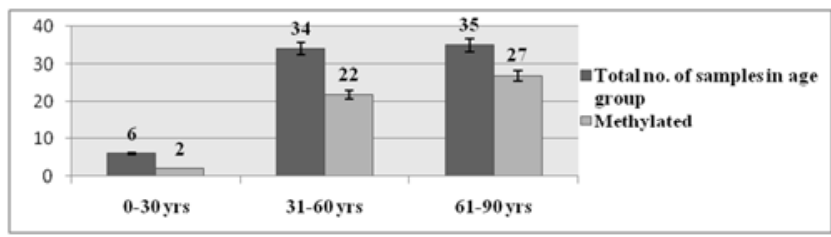

Graph 5. Methylation Pattern of Different Age Groups in samples.

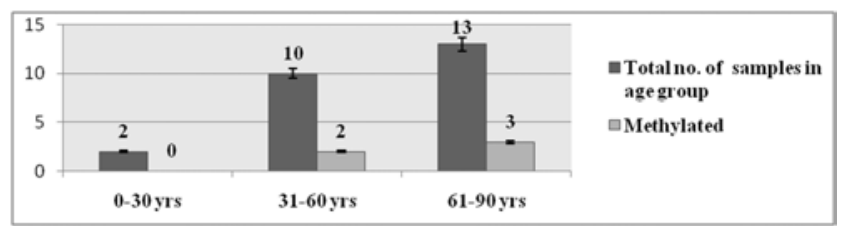

Graph 6. Methylation Pattern of Different Age Groups In Normal samples.

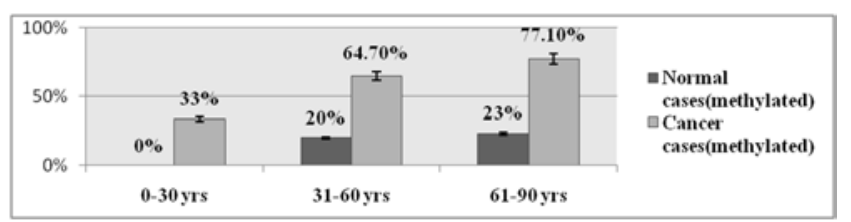

Graph 7. Frequency of Promoter Methylation of Different Age Groups intheHistopathologically Confirmed Cancer \& Normal Cases.

\section{Discussion}

Epigenetic mechanisms, such as hypermethylation of CpGIsland of promoter regions, have been proposed as a mechanism of gene inactivation in tumour cells (Esteller et al., 2002). Our study showed methylated BRCAlassociation of promoter methylation between breast cancer and histologically confirmed normal controleswas found to be significant which was supported bythe findings of Catteau et al., 1999; Esteller et al., 2008; Niwa et al., 2000; Rice et al., 2000, but our results are incontradictic with the findings of Knudson et al., 1971, Knudson et al., 1985 as thy showed in their results that BRCA1 promoter hypermethylation is not involved in the breast cancer. Our results are showing that BRCA1 promoter hypermethylation was involved in breast carcinoma as an epigenetic change. Our study also supports the findings of Hanahan D. et al., 2000 as they studied that tumour of breast cancer patients showed promoter hypermethylation of BRCA1 gene, these results were similar to our studied reults, that in BRCA1 gene in breast cancer patients, there is an altrationsof methylation pattern, which may the causative agent for the breast cancer and is also in support of the findings of Friedman et al., 1994; Easton 1997, Castilla et al., 1994 they studied in their study that alterations in BRCA1 have been estimated to be responsible for about $50 \%$ of familial breast cancer. Our findings was also supported by several workers in different parts of the world in their studies reportes that aberrant promotor hypermethylationof BRCA1 could occur in breast carcinoma, as they observed during their studies that in some breast cancer cell lines and primary tumors. (Rice., et al, 18. Magdinier et al., 1998 and Catteau et al., 1999)

Promoter hypermethylation of BRCA1 gene in breast cancer patients in our study based on comparison to the worldwide researches and the mean of frequency of hypermethylated BRCA1 was $68 \%$ of the breast cancer tissue samples showed methylated $B R C A-1$ promoter and $32 \%$ of the cases however showed unmethylated $B R C A-1$ promoter. Almost all $80 \%$ of the histopathologically confirmed normal tissue samples showed unmethylated $B R C A-1$ promoter, except only in $20 \%$ normal cases where $B R C A-1$ promoter was found to be methylated and was rather higher than Tapia et al. (2008) and Phuong Kim Truong et al. (2013), (19,20). Regarding to clinical parameters, in our study, When the frequency of $B R C A-1$ promoter methylation was compared with clinical staging of the disease, $B R C A-1$ promoter methylation was found to be certainly higher in Stage III/IV (75\%) compared to Stage I/ II (62.7\%) but the difference was not statistically significant $(\mathrm{P}=0.0674)$. The frequency of promoter methylation was found $33.33 \%$ in age group (0-30 yrs), $64.7 \%$ in age group (31-60 yrs) and $77.1 \%$ in age group (61-90) yrs)which was in support of the findings of Phuong Kim Truong et al. (2013), Hora Loghmani et al. (2014) as in there studies there was no any significant differences between the hypermethylated BRCA1 promoter gene and the patient ages, tumor grades and stages.

Our study confermes that BRCA1 promotor region was specific characteristic of breast cancer patients in ethenic population of Kashmir valley, but in more additional studies are needed to understand the nature of association of promotor hypermethylationin breast cancer, so the epigenetic methylation may be used as early diagnostic and proganostic tools in breast cancer patients. 


\section{Conclusion}

This study has interestingly revealed that promoter region hypermethylation status of mismatch repair gene BRCAI shows a significant increase in Breast cancer patients of Kashmiri origin as compared to controls. This became more apparent when the data for hypermethylation was interpreted taking Clinical stage into consideration and it was seen here that stage III/IV shows higher frequency of promoter region hypermethylation as compared to stage I/II which was earlier reported in literature Further it has been seen that also patients of above 60 years of age shows high frequency compared to below 60 years of age and breast carcinogenesis is a stepwise process of the accumulation of genetic and epigenetic abnormalities. It is clear that promoter hypermethylationis important for multistep process though genetic changes in the progression of breast carcinogenesis. Our study has supplemented the steadily growing list of genes inactivated by promoter hypermethylation in breast carcinoma and has provide not only new insights into the molecular basis of the diseases but also list of interesting candidate genes for the development of molecular markers which might contribute to the improvement of diagnosis. From our results it was confermed that BRCA1 promotor region was specific characteristic of breast cancer patients in ethenic population of Kashmir valleywas associated with breast cancer risk.

\section{Referances}

[1] AurelieCatteau and Joanna R. Morris.Cancer biology. 2002, 12: $359-371$.

[2] Castilla LH, Couch FJ, Erdos MR, Hoskins KF, Calzone K, Garber JE. Mutations in the BRCA1 gene in families with early-onset breast and ovarian cancer. Nat Genet. 1994; 8:387-91.

[3] Catteau A, Harris WH, Xu CF, Solomon E. Methylation of the BRCA1 promoter region in sporadic breast and ovarian cancer: correlation with disease characteristics. Oncogene. 1999; 18:1957-65.

[4] Easton D. Breast cancer genes, what are the real risks? Nat Genet. 1997; 16:210-1.

[5] Esteller M (2002). CpG island hypermethylation and tumor suppressor genes:a booming present, a brighter future. Oncogene. 2002; 21: 5427-40.

[6] Esteller M., Epigenetics in cancer,N. Engl. J. Med. 2008; 3 (58): 1148-1159.

[7] Fang WJ, Zheng Y, Wu LM, et al. Genome-wide analysis of aberrant DNA methylation for identification of potential biomarkers in colorectal cancer patients. Asian Pac J Cancer 2012.
[8] Friedman LS, Ostermeyer EA, Szabo CI, Dowd P, Lynch ED, Rowell SE, King MC. Confirmation of BRCAI by analysis of germline mutations. Nature genetics. 1994; 8: 399-404.

[9] Hanahan D, Weinberg RA. The hallmarks of cancer. Cell. 2000; 100 (1): 57-70.

[10] Hora Loghmani, MehrdadNoruzinia, Hossein Abdul-Tehrani, et al. Association of estrogen receptors' promoter methylation and clinicopathological characteristics in Iranian patients with breast cancer. Molecular and Biochemical diagnosis (MBD). 2014; 1(1): 21-33.

[11] Knudson AG. Hereditary cancer, oncogenes, and antioncogenes. Cancer Research,. 1985; 45(4): 1437-1443.

[12] Magdinier F, Ribieras S, Lenoir GM, Frappart L, Dante R. Down-regulation of BRCA1 in human sporadic breast cancer; analysis of DNA methylation patterns of the putative promoter region. Oncogene.1998; 17 (24): 3169-76.

[13] Niwa Y, Oyama T, Nakajima T. BRCA1 expression status in relation to DNA methylation of the BRCA1 promoter region in sporadic breast cancers. Cancer Science. 2000; 91 (5): 51926.

[14] Parvin JD. Overview of history and progress in BRCA1 research: the first BRCA1 decade. Cancer biology \& therapy. 2004; 3 (6): 505-8.

[15] Phuong Kim Truong, ThuanDuc Lao, Thao Phuong Thi Doan, Thuy Ai Huyen Le1. BRCA1 Promoter Hypermethylation Signature for Early Detection of Breast Cancer in the Vietnamese Population. Asian Pac J Cancer Prev. 2014; 15 (22): 9607-9610.

[16] Phuong Kim Truong, ThuanDuc Lao1, Thao Phuong Thi Doan, et al. BRCA1 Promoter Hypermethylation Signature for Early Detection of Breast Cancer in the Vietnamese Population. Asian Pac J Cancer Prev. 2014; 5 (22): 96079610.

[17] Ramezani F, Salami S, Omrani MD, Maleki D. CpG island methylation profile of estrogen receptor alpha in Iranian females with triple negative or non-triple negative breast cancer: new marker of poor prognosis. Asian Pac J Cancer Prev. 2012; 13: 451-7.

[18] Rice JC, Ozcelik H, Maxeiner P, Andrulis I, Futscher BW. Methylation of the BRCA1 promoter is associated with decreased BRCA1 mRNA levels in clinical breast cancer specimens. Carcinogenesis. 2000; 21 (9): 1761-5.

[19] Showkat Ahmad Bhat, Manzoor R Mir, Sabhiya Majid. Serum lipid profile of breast cancer patients in Kashmir. J Invest Biochem. 2013; 2 (1): 26-31.

[20] Tapia T, Smalley SV, Kohen P, et al. Promoter hypermethylation of BRCA1 correlates with absence of expression in hereditary breast cancer tumors. Epigenetics. $2008 ; 3,157-63$.

[21] Wernberg JA, Yap J, Murekeyisoni C, Mashtare T, Wilding GE. Multiple primary tumors in men with breast cancer review. J SurgOncol. 2009; 99: 16-9. 